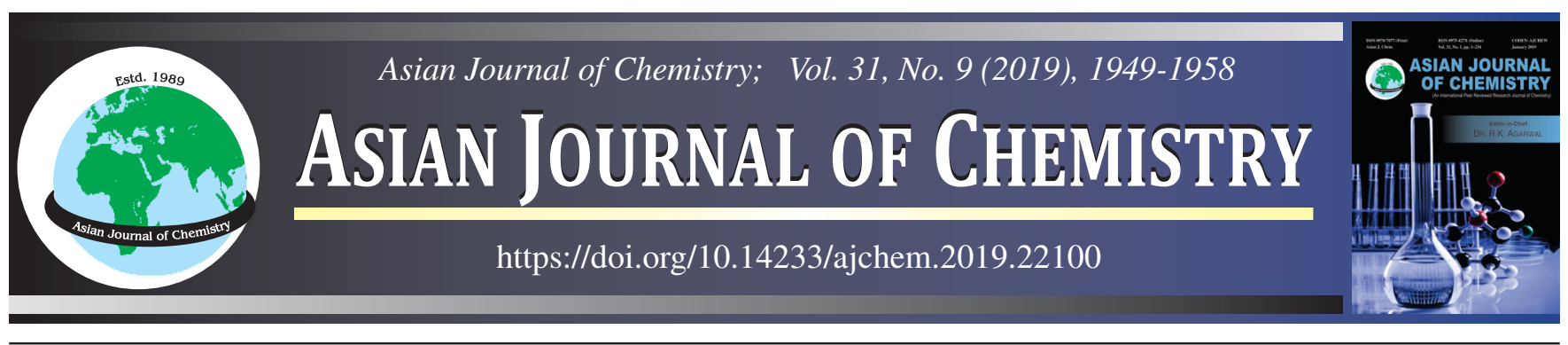

\title{
Probing into Methylene Blue Interaction with Polyglutamic Acid: Spectroscopic and Molecular Dynamics Simulation Studies
}

\author{
Poonam Mishra Chatterjee ${ }^{1,2, \dagger}$, Deepika Pandey Tiwari ${ }^{1,2, \dagger}$, Silpi Datta $^{2, \bullet}$, \\ Suman Chakrabarty ${ }^{3, \bullet}$, Ritu Raval ${ }^{1, \bullet}$ and Ashok Kumar Duber, ${ }^{2, *}$
}

${ }^{1}$ Department of Biotechnology, Manipal Institute of Technology, Manipal Academy of Higher Education, Manipal-Karnataka-576104

${ }^{2}$ Department of Food Science and Technology, Tata Chemicals Ltd-Innovation Centre, Survey Number 315, Hissa Number 1-4, Ambedveth, Paud Road, Mulshi, Pune-412111, India

${ }^{3}$ School of Chemical, Biological \& Macromolecular Sciences, S. N Bose National Centre for Basic Sciences, Salt Lake, Kolkata-700106, India

$\dagger$ These authors contributed experimental work equally

*Corresponding author: Tel: +91 20 66549707; E-mail: adubey@ tatachemicals.com

Received: 28 March 2019;

Accepted: 14 April 2019;

Published online: 31 July 2019;

AJC-19481

Polyglutamic acid (PGA) is an anionic biopolymer which is stained with methylene blue (MB) in agar or polyacrylamide gels for analysis. Polyglutamic acid identification involves a tedious extraction and analytical process. The molecular association of polyglutamic acid and methylene blue can be used for rapid spectroscopic detection of polyglutamic acid production during fermentation. This triggered the study on investigation of polymer-dye interaction mechanism. Concentration range of polyglutamic acid, 0.001 to $0.06 \mu \mathrm{M}$ with a fixed methylene blue concentration of $25 \mu \mathrm{M}$ exhibited significant differences in the spectra. Preferential higher order aggregate formation of methylene blue molecules was substantiated with molecular dynamics simulation results. Fluorescence spectroscopy demonstrated a quenching effect of polyglutamic acid on methylene blue fluorescence until a certain concentration range, beyond which the SternVolmer (SV) plot shows a negative deviation. Polyglutamic acid was observed to promote higher order of intramolecular stacking interactions of methylene blue, possibly due to interplay of electrostatic and hydrophobic interactions between polyglutamic acid, methylene blue alone and PGA-MB systems.

Keywords: Polyglutamic acid, Methylene blue, Dye, Spectrophotometry.

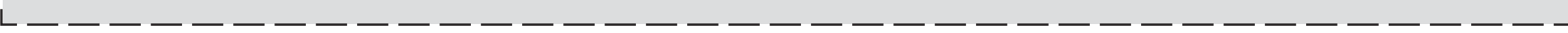

\section{INTRODUCTION}

Polymeric materials have gained a lot of importance globally in the past few decades due to their innumerable applications in almost every aspect of our existence. However synthetic polymers obtained from non-renewable sources have negative impact on the environment due to their limitation of recyclability. This awareness for development of sustainable environment has led to an exploration of new generation of compounds called biopolymers. Polyglutamic acid is one such biopolymer that is made up of repeated units of glutamic acid (GA). This polyamino acid can also be produced synthetically, but due to its lower molecular weight has limited applications [1]. Bacterially produced polyglutamic acid has higher molecular weight ranging from 100 to $100,000 \mathrm{kDa}$ [2]. Polyglutamic acid is versatile in its applications ranging from controlled drug delivery to food and beverage [3], waste water treatment and skin care.

There has been an extensive research carried out in the area of bacterial production of polyglutamic acid. A major emphasis has been done in the screening of the microorganisms producing polyglutamic acid. Currently reported methods of screening along with detection of microbially produced polyglutamic acid though effective, are tedious and time consuming [4]. Moreover, most of these methods involve chemical treatments with minerals acids or salts [5]. Some of them have also reported the use of complex instrumentation like NMR [6]. In earlier paper, we had reported a rapid screening protocol for $\gamma$-polyglutamic acid producing microorganisms using methylene blue dye in solid agar media and an attempt was

This is an open access journal, and articles are distributed under the terms of the Attribution 4.0 International (CC BY 4.0) License. This license lets others distribute, remix, tweak, and build upon your work, even commercially, as long as they credit the author for the original creation. You must give appropriate credit, provide a link to the license, and indicate if changes were made. 
made to detect polyglutamic acid production in aqueous medium using the fermentation broth directly [7]. To our best of knowledge, this was also the first study to extend the application of PGA-MB interaction to differentiate between the glutamic acid and polyglutamic acid [7]. Spectroscopic detection of polyglutamic acid has been reported earlier [8], however the distinction between glutamic acid and polyglutamic acid becomes essential in presence of glutamic acid in fermentation broth, for which the use of dye makes the process of detection easier. There have also been reports of using methylene blue dye for staining of SDS-Page for detection of polyglutamic acid [9]. Adsorption capability of methylene blue onto polyglutamic acid has also been reported [10]. Though some of the previous reports have revealed that an electrostatic binding is responsible between polyglutamic acid and methylene blue, very few articles have elucidated the mechanism of their binding [11]. Therefore, in the present study, we have made an attempt to probe into the interaction mechanism of a higher molecular weight polyglutamic acid with methylene blue in aqueous medium. Consequently, the study also reports changes occurring in the behaviour of methylene blue as a result of PGA-MB interaction. Impacts of external factors capable of influencing the complex formation were also studied through systematic probing using absorption and fluorescence properties of the complex. It was important to explore if the staining of the macromolecule was the result of charge-charge interactions only or if the polymerdye binding was promoted or affected by the concentrations of both the molecules.

Methylene blue is a dye containing basic thiazine group, which is used preferably as a redox indicator due to the existence of equilibrium between its reduced and oxidized states [12]. It is also known to be a metachromatic dye which shows change in colour depending on environmental conditions. The spectra of aqueous solution of methylene blue reveal the presence of an intense absorption band of monomeric and a small shoulder like appearance of dimeric forms under neutral and acidic conditions [13]. Methylene blue and methylene blue like dyes have been reported to interact with various polyelectrolytes and polyanions [14]. There have been earlier reports of changes in the aggregate forms of the dye under the influence of various environmental conditions [15]. The above mentioned difference in the aggregation tendency can be utilized to detect the presence of polyanions such as polyglutamic acid. The major factors involved in aggregation phenomenon are reported to be hydrophobic forces and the effects of structural features of water. $\pi$ - $\pi$ stacking interactions between the aromatic groups have also been reported to occur in the aggregated form of the dye $[16,17]$.

There are several reports on the aggregation patterns of methylene blue under various conditions such as $\mathrm{pH}$, temperature, ionic strength, etc. which lead to spectral shift in the absorbance of the dye $[8,18]$. This metachromatic property of the dye is attributed to the binding of dye molecules with the counter ions in its vicinity as a result of electrostatic and hydrophobic interactions [19]. Further analysis of the effect of conformational changes occurring in methylene blue and polyglutamic acid in the bound and free forms was carried out. We have focused our study on understanding the mecha- nism of binding of these two molecules in both solid and liquid media. The capacity of methylene blue binding with polyglutamic acid can be exploited to establish a rapid detection method for polyglutamic acid production during fermentation.

\section{EXPERIMENTAL}

Dyes, namely, rhodamine B (RB), bromocresol green $(\mathrm{BG})$, acridine orange $(\mathrm{AO})$ and methylene blue were procured from Himedia, neutral red (NR) from Fisher Scientific was used for all the UV-visible experiments. Later methylene blue was assessed for polyglutamic acid binding using other analytical tools. Polyglutamic acid from Hebei-fulong with molecular weight $1550 \mathrm{kDa}$ with polydispersity of 1.4 was used for the analysis. Deionized water passed through $0.2 \mu \mathrm{m}$ filter was used to make $200 \mu \mathrm{M}$ stock solutions of the dye and $0.6 \mu \mathrm{M}$ polyglutamic acid stock solutions. Urea from Sigma was sourced for UV-visible based experiments All the experiments were performed in deionized water passed through 0.2 $\mu \mathrm{m}$ filter at temperature $25^{\circ} \mathrm{C}$ and $\mathrm{pH} \sim 7$ using various analytical tools.

UV-visible spectroscopy: Dye selection was carried out using UV-visible NIS Varian Cary 5000. Later UV-visible Spectrophotometer model-Shimadzu UV-1800 was used for all the absorption based spectra of methylene blue based solutions. Quartz cuvettes with the capacity of $1.4 \mathrm{~mL}$ and $10 \mathrm{~mm}$ path length were used for analysis of $1 \mathrm{~mL}$ samples. Water was used as the reference blank in all the experiments and the baseline was run for the same. UV-visible Scans were taken in the wavelength range of 190 to $800 \mathrm{~nm}$ using UVProbe 2.43 software. $10 \mathrm{~mL}$ solutions of $25 \mu \mathrm{M}$ methylene blue and MBPGA complex with varying concentration range of polyglutamic acid were prepared throughout the experiments.

Fluorescence spectroscopy: Polyglutamic acid in the range of 0.0006 to $0.06 \mu \mathrm{M}$ was allowed to react with three different concentrations of methylene blue in the range of 6.25 to $25 \mu \mathrm{M}$. Cary Eclipse Fluorescence Spectrophotometer was used for emission spectroscopy in the wavelength range of $550 \mathrm{~nm}$ to $1100 \mathrm{~nm}$ with excitation wavelength of $550 \mathrm{~nm}$.

Molecular dynamics (MD) simulations: All MD simulations were performed using Gromacs 5.0.7 software [20] with Amber99SB-Ildn force field [21] and TIP3P water model [22]. All the structures were solvated into cubic boxes with periodic boundary conditions. The box sizes were set to ensure a distance of at least $1 \mathrm{~nm}$ between the protein (polyglutamic acid) and the box boundaries. Adequate numbers of $\mathrm{Na}^{+}$and $\mathrm{Cl}^{-}$ions were added to neutralize the charged system as well to maintain $150 \mathrm{mM}$ salt concentration. The system was energy minimized using steepest descent algorithm. All the systems were equilibrated for $2 \mathrm{~ns}$ in NPT ensemble using a velocity rescale thermostat [23] and Parrinello-Rahman barostat [24] at $300 \mathrm{~K}$ and 1 bar. Long-range electrostatic interactions were calculated with the particle mesh Ewald (PME) summation method [25] with a grid spacing of $0.16 \mathrm{~nm}$ and fourth-order cubic interpolation. For short range electrostatics and van der Waals interaction, a cut-off distance of $1 \mathrm{~nm}$ was used. All covalent bonds were constrained using the LINCS algorithm [26]. The integration time step was set to $2 \mathrm{fs}$. The production run was further continued for $20 \mathrm{~ns}$ and coordinates were saved every $2 \mathrm{ps}$. 


\section{RESULTS AND DISCUSSION}

Dye selection for screening polyglutamic acid producing microbes: Dye selection for isolation of polyglutamic acid producing strains was a crucial step in the primary screening protocol establishment. We have screened various dyes and $\mathrm{pH}$ indicators to selectively distinguish polyglutamic acid producers from the mixed flora of microorganisms. Several dyes were assessed for their binding capacity with polyglutamic acid to selectively differentiate between polyglutamic acid producers from non-producers as mentioned in previous studies [6]. Solid media containing methylene blue (MB), neutral red (NR) and rhodamine $B(\mathrm{RB})$ exhibited a zone of clearance indicative of possible interactions of dye with secreted polyglutamic acid [26] and/or possible methylene blue adsorption on polyglutamic acid [28]. To further elucidate the specificity of methylene blue for polyglutamic acid beyond solid media, we expanded our studies to aqueous medium. Two more molecules i.e. acridine orange (AO) and bromocresol green (BG) were added to the list of the above dyes to give a more comprehensive picture of the structural requirements. Acridine orange has already been reported to bind to polyglutamic acid exhibiting cotton effects [29]. All the above four dyes (methylene blue, neutral red, rhodamine B and bromocresol green) have a basic molecular framework consisting of tricyclic ring. The molecular structures of all five dyes are shown in Fig. 1 where the carbon atom opposite to the nitrogen atom in the central ring of acridine orange is replaced by a sulphur atom in methylene blue, a nitrogen atom in neutral red and a benzaldehydic group in rhodamine $\mathrm{B}$. The nitrogen atom in acridine orange has been replaced by an oxygen atom in rhodamine B. Neutral red also has an unsubstituted amine group and an extra methyl substitution in one of the terminal rings. Acridine orange, neutral red and methylene blue are the meta-chromatic dyes with simple planar structures. Apart from these bromocresol green having a three ring non planar structure was also selected to emphasize the difference of the fused ring structure exhibiting metachromatic effect. All these molecules were investigated for their binding capacity with polyglutamic acid in liquid media using UV-visible spectroscopy.
Acridine orange, neutral red, methylene blue and rhodamine B are known to undergo self-association [30] due to fused planar framework, but there are no such evidences available for the similar phenomenon in bromocresol green due to steric hindrances. An utmost care was taken to execute the experiments with in the spectral range of measurable absorbance i.e. OD 0.1 to 1 . The spectral shifts were more prominent in methylene blue (Fig. 2a) as compared to acridine orange (Fig. $2 b)$. UV-visible spectra of methylene blue with polyglutamic acid showed the prominence of binding. The dyes like rhodamine B (Fig. 2c) and bromocresol green (Fig. 2d) didn't show any significant visible shift in the absorption spectra of the respective dyes in presence of $0.003 \mu \mathrm{M}$ polyglutamic acid. In rhodamine B and bromocresol green, though, the difference in the absorbance intensity peak was observed in presence of glutamic acid and polyglutamic acid but both could not be differentiated. Neutral red $\mathrm{R}$ having significance in the screening of polyglutamic acid producing microorganisms on solid agar plate was also assessed for its capability to bind with poly-glutamic acid molecule. UV-visible studies with neutral red as shown in Fig. 2e were not conclusive enough to differentiate between monomer and polymer form of glutamic acid units due to lack of spectral shifts. Though, rhodamine B showed an increase in the intensity of the absorbance in presence of $0.003 \mu \mathrm{M}$ polyglutamic acid but even $100 \mathrm{mM}$ glutamic acid had no effect on it. Similarly, when bromocresol green was used as an indicator dye, the difference between monomer and polymer could not be observed as both glutamic acid and polyglutamic acid appeared to interact with the dye in same way. However, significant spectral shifts were observed in case of methylene blue and acridine orange as shown in Figs. $2 \mathrm{a}$ and $2 \mathrm{~b}$, respectively.

Neutral red has been studied with respect to zone of clearance and molecular weight analysis in solid media [8]; however, not much spectroscopic studies have been reported on the application of above mentioned dyes. In present UV-visible spectroscopic studies, we found that neutral red also binds to polyglutamic acid but the change in spectra was more evident in PGA-MB complex than neutral red in liquid media. Acridine orange and methylene blue exhibited better effect in spectral<smiles></smiles><smiles>Cc1c(C2(c3cc(Br)c(O)c(Br)c3C)OS(=O)(=O)c3ccccc32)cc(Br)c(O)c1Br</smiles><smiles>CC(C)c1ccc2cc3ccc(N(C)C)cc3nc2c1</smiles>

b<smiles>Cc1cc2nc3ccc(N(C)C)cc3nc2cc1N</smiles><smiles>CCN(CC)c1ccc2c(-c3ccccc3Cl)c3cc(C)c(=[N+](CC)CC)cc-3oc2c1</smiles>

c

Fig. 1. Molecular structure of a) methylene blue b) acridine orange, c) rhodamine B, d) bromocresol green, e) neutral red 

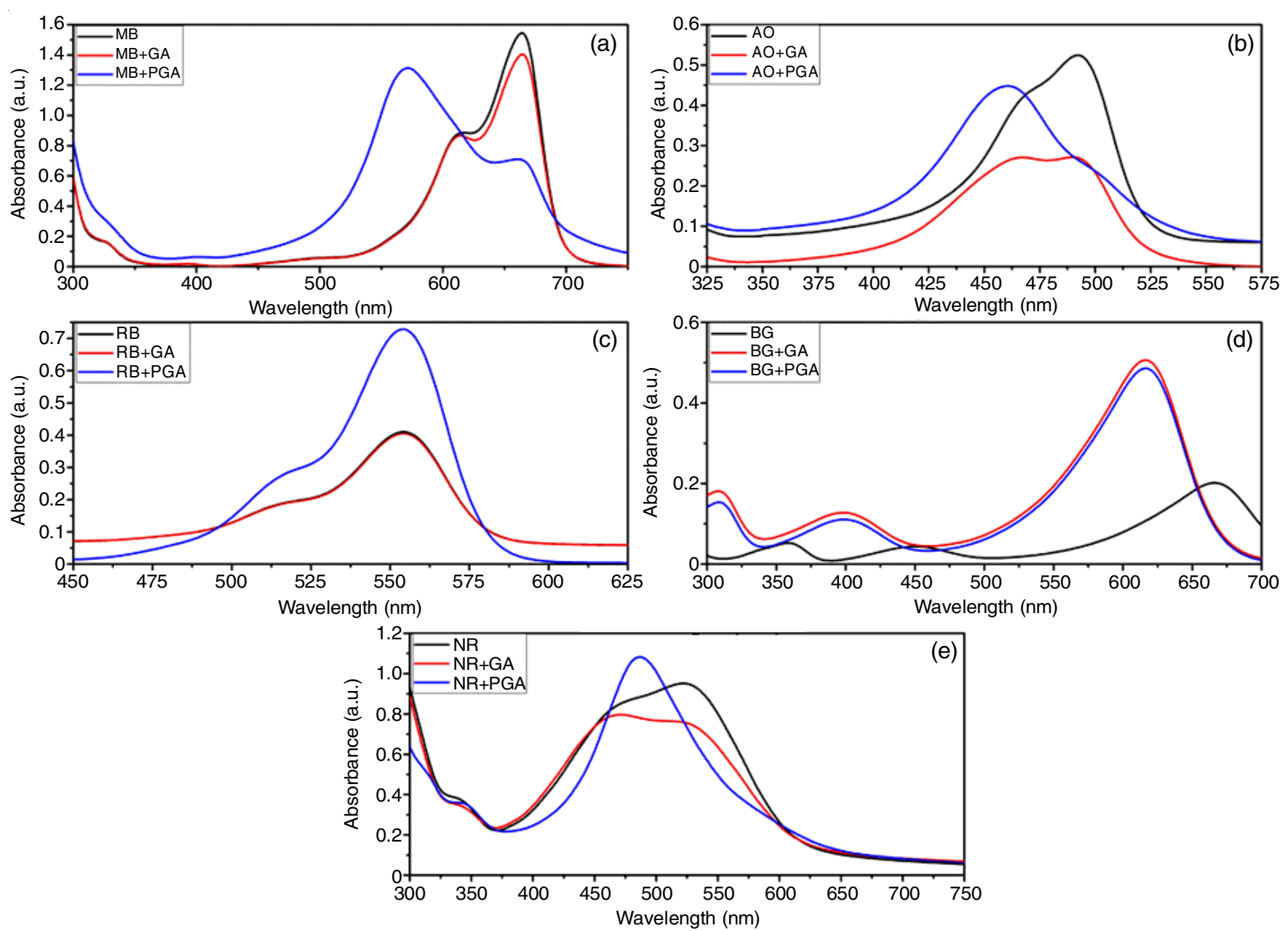

Fig. 2. UV-visible absorbance spectra in the respective presence of $0.003 \mu \mathrm{M}$ polyglutamic acid and $100 \mathrm{mM}$ glutamic acid along with (a) methylene blue (b) acridine orange (c) rhodamine B (d) bromocresol green (e) neutral red

shift than other dyes; hence further studies were carried forward using these two dyes.

UV-visible spectroscopy: Role of polyglutamic acid in dye aggregation: Aqueous solution of methylene blue in the concentration range between 1-40 $\mu \mathrm{M}$ occur in monomer and dimer forms in aqueous solution [17]. The UV-visible spectra of methylene blue (Fig. 2a) in an aqueous medium exhibited absorption maxima with a shoulder of dimer at 610 and monomer at $664 \mathrm{~nm}$ respectively that are in accordance with the previous reports $[12,31,32]$. The UV-visible spectra of aqueous solution of polyglutamic acid however show peaks in the UV region only at $216 \mathrm{~nm}$ that does not hinder the prominent peaks of methylene blue. In presence of polyglutamic acid (concentration range 0.0006 to $0.06 \mu \mathrm{M}$ ) as shown in Fig. 3, UV-visible spectra of $25 \mu \mathrm{M}$ methylene blue exhibits another peak at 571 $\mathrm{nm}$ indicating a higher order of oligomerization [33]. The peak at $571 \mathrm{~nm}$ increases whereas the monomer peak at $664 \mathrm{~nm}$ significantly decreases with the increasing polyglutamic acid concentration. The dimer peak at $610 \mathrm{~nm}$ gets masked by the increase in the peak height at $571 \mathrm{~nm}$. A lower concentration of polyglutamic acid has a greater affinity to bind to methylene blue molecules in trimeric configuration. At the concentration of $0.003 \mu \mathrm{M}$ of polyglutamic acid we observed the maximum absorbance of methylene blue molecules at $571 \mathrm{~nm}$ and on further increasing the concentration of the polymer, the peak at $571 \mathrm{~nm}$ reduced in size and gradually merged into the $610 \mathrm{~nm}$ showing a probable increase in the dimeric form of methylene blue. This change in the spectra did not lead to the formation of monomers completely when compared with aqueous solution of methylene blue, rather, the spectra was observed to enhance both the dimer and monomer peaks simultaneously as evident from Fig. 3. This indicated the impact of presence of polyglutamic acid in the solution on the aggregate formation of methylene blue molecules.

A similar phenomenon was also reported by Moore et al. [34], where they mentioned that in presence of few anionic sites i.e. at very low concentration of polymer, the methylene blue molecules have a tendency to form trimer as a consequence of fewer number of anionic sites for binding [34]. The overlapping of monomer and dimer with the trimers peaks in the absorption spectra of methylene blue indicate the existence of various aggregate forms concomitantly. The presence of forces like van der Waals and hydrophobic interactions provides thermodynamic stability to the stacked pattern existing between the aromatic rings of methylene blue [23]. However, the stacking between the aromatic rings of methylene blue molecules gets perturbed due to the interaction between carboxylate anion which is a negatively charged moiety in polyglutamic acid with the positively charged nitrogen centre of methylene blue [28]. The hydrophobic interaction between the aromatic 


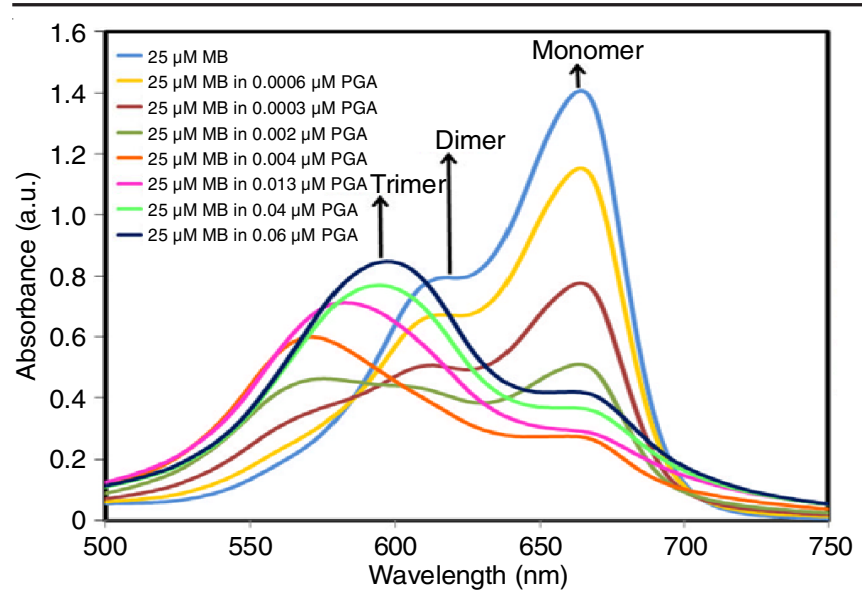

Fig. 3. UV-visible absorption spectra of $25 \mu \mathrm{M}$ methylene blue alone and in combination with polyglutamic acid in the concentration range of 0.0006 to $0.06 \mu \mathrm{M}$. Absorption spectra exhibited a distinct formation of trimers with polyglutamic acid as observed under UVvisible spectroscopy

rings of methylene blue assists it to remain in the dimeric state in water however the dye undergoes a structural change in the presence of polyglutamic acid. It is already reported that polyanions trigger the formation of higher ordered aggregates in dye [26]. PGA-MB binding can thus be a result of electrostatic interaction between carboxylate group of polyglutamic acid and nitrogen centre of dye.

The usual presence of methylene blue in its monomer and dimer forms at lower concentrations tends to get affected under different environmental influences [12]. It is a cationic dye that on binding with anionic groups or charges in its vicinity leads to its aggregation [29]. Coates [35] has reported the phenomenon of aggregation of dye molecules under the influence of ionic strength of the solutions. However, in our studies the gradual addition of $\mathrm{NaCl}$ to the PGA-MB complex led to reduction in the absorbance (peak height) of trimer region at $571 \mathrm{~nm}$ and enhancement of dimer and monomer absorbance peaks (Fig. 4). Thus, at high ionic strength we observed an enhancement in the formation of dimers. This indicates that at low salt concentration, the methylene blue molecules in the vicinity of polyglutamic acid surface experience enhanced hydrophobic interactions due to increased stacking between the aromatic rings of positively charged methylene blue mole-

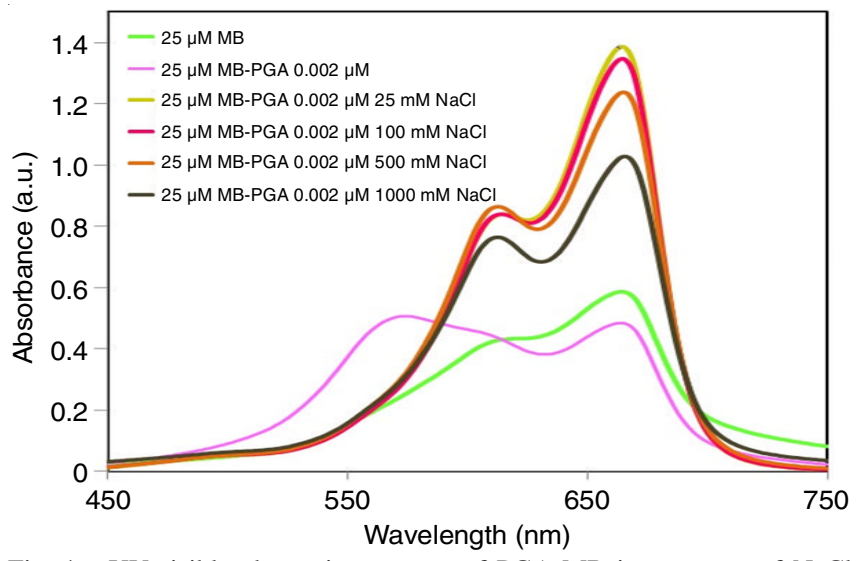

Fig. 4. UV-visible absorption spectra of PGA-MB in presence of $\mathrm{NaCl}$ showing conversion of higher ordered aggregates of methylene blue to their respective dimers and monomer cules. On the contrary, the effect of electrostatic interaction reduces between polyglutamic acid and methylene blue on addition of salt in the solution because of the presence of positive counter ions $\left(\mathrm{Na}^{+}\right)$. Methylene blue molecules get separated from polyglutamic acid and the population of lower order aggregates like formation of monomers and dimers from trimers becomes predominant.

This study was in accordance with the research work by Hu et al. [36] where they reported that increased ionic strength caused by addition of $\mathrm{Na}^{+}$and $\mathrm{Cl}^{-}$ions tend to reduce the electrostatic interactions. Therefore polyglutamic acid, if present, may act as a mild polyelectrolyte that on binding with methylene blue causes trimerization and formation of higher ordered aggregates of methylene blue where an additional absorption maxima shows up at $571 \mathrm{~nm}$ representative of trimer form of methylene blue. A similar experiment was carried to check the existence of higher order oligomers of methylene blue in presence of glutamic acid and sodium chloride as well. However, there was no such evidence of trimer formation in the spectral data of methylene blue with glutamic acid and $\mathrm{NaCl}$ that further helped to infer the existence of the electrostatic interactions of methylene blue with the bulky polyglutamic acid facilitates the aggregation of dye molecules.

Metachromasy of dye in presence of polyglutamic acid: In the UV-visible studies we have observed the phenomenon of dye aggregation of methylene blue on interaction with polyglutamic acid molecules and this effect of dye aggregation could be visually perceived as well due to colour change of the solution. This colour change was visible in the concentration range of polyglutamic acid from 0.0012 to $0.006 \mu \mathrm{M}$ in presence of $25 \mu \mathrm{M}$ methylene blue that exhibited the metachromatic behaviour of the dye. It was observed that the blue colour of methylene blue changed to purple on addition of lower concentrations of polyglutamic acid but on increasing the concentration of the biopolymer in the medium, the colour of methylene blue changed back to blue. Delocalization of electrons on the aromatic rings of methylene blue was majorly responsible for the change in the metachromasy of the dye.

Methylene blue undergoes reversal in metachromasy on being diluted, but similar phenomenon was not observed under the higher concentration range of polyglutamic acid i.e. from 0.006 to $0.06 \mu \mathrm{M}$ as shown in Fig. 5. There was no evidence of trimer to monomer conversion and the aggregation of the dye was restricted to dimer species only, even after increasing the concentration to $6 \mu \mathrm{M}$ of polyglutamic acid. The findings were in concurrence with the existing studies on the dye where the researchers have reported aggregation change with respect to polydispersity of polyanions [26]. We hereby report that the interaction between polyglutamic acid and methylene blue is concentration dependent; apart from the charge-charge interactions where the polyglutamic acid molecules on addition to methylene blue bound water molecules perturb the self-association of the dye. The existence of dimers and the pattern of stacking suggest the de tion of the above mentioned dye from the Beer and Lambert's law [37]. The binding constant for the equilibrium could not be calculated as we could not obtain the isosbestic point in the spectra indicating the involvement of more than one type of binding. Similar type of studies were 


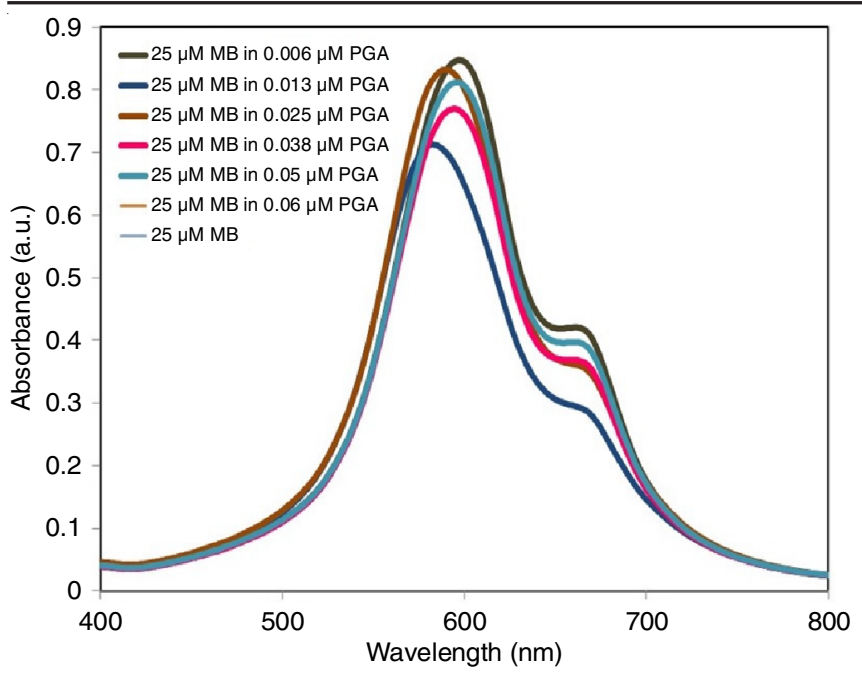

Fig. 5. Comparison of UV-visible absorption spectra of $25 \mu \mathrm{M}$ methylene blue alone with higher concentrations of polyglutamic acid ranging from 0.006 to $0.06 \mu \mathrm{M}$ in presence of methylene blue. No shift in the spectra at high concentration of polyglutamic acid was observed

carried out where binding of DNA-MB complex did not exhibit any isosbestic point due to prevalence of more than one type of binding [38].

Therefore, the presence of polyglutamic acid was observed to promote the aggregation of methylene blue with the majority of trimer species as evident from the results were again in alignment with our UV-visible spectral observations.

Urea breaker experiment: The involvement of hydrophobic and electrostatic interactions between methylene blue and polyglutamic acid, was further analyzed using urea in the solution containing the mix. The PGA-MB spectral studies were performed in the presence of $5.5 \mathrm{M}$ aqueous solution of urea. The red shift in the spectra and increase in the intensity of monomeric peak at $664 \mathrm{~nm}$ as shown in Fig. 6 exhibited the effect of urea on PGA-MB complex formation in aqueous solution.

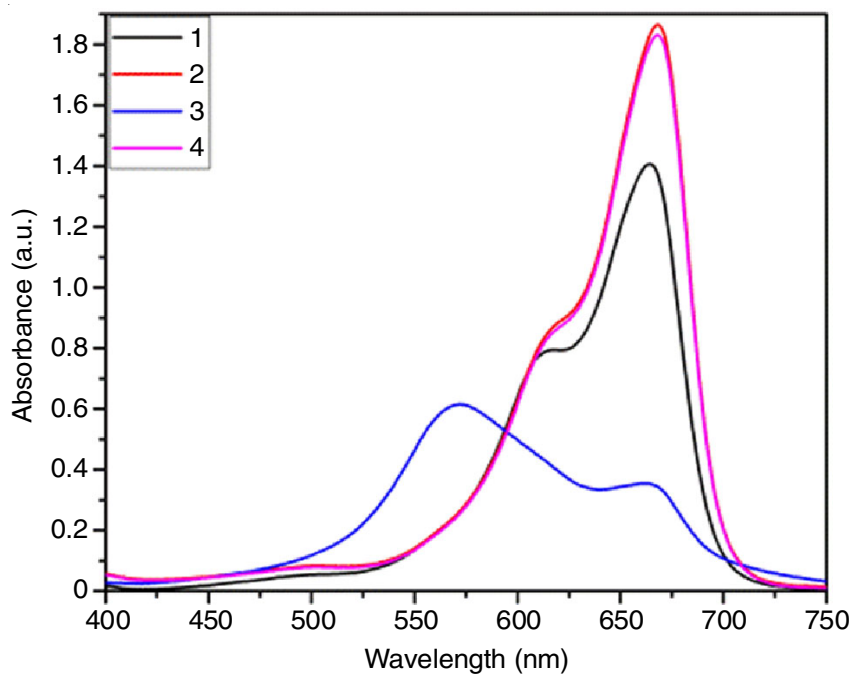

Fig. 6. UV-visible absorption spectra illustrating the effect of urea on methylene blue in presence of polyglutamic acid. The symbols represent (1-25 $\mu \mathrm{M}$ methylene blue, 2- $25 \mu \mathrm{M}$ in presence of $5.5 \mathrm{M}$ urea, 3- $0.003 \mu \mathrm{M}$ polyglutamic acid bound with $25 \mu \mathrm{M}$ methylene blue, $4-0.003 \mu \mathrm{M}$ polyglutamic acid in presence of $25 \mu \mathrm{M}$ methylene blue and $5.5 \mathrm{M}$ urea)
Urea is known to be an efficient molecular structure breaker and chemical denaturant for proteins $[39,40]$. There are several hypotheses on the mechanism of action of urea on the tertiary structure of proteins including breakdown of water structure, preferential solvation of hydrophobic residues and adsorption of the urea on to the surface of proteins leading to swelling and denaturation. The dye rearranges itself in the water solution once the bond between PGA-MB breaks in presence of urea. We have observed that urea not only disturbs the self-association of dye in water arising due to hydrophobic interactions but it also perturbs the molecular association of polyglutamic acid and methylene blue. The changes associated due to presence of urea probably lead to increase in the surface tension of water molecules and its dielectric constant is recognized to cause structural defects in the self-association of dye molecules [41]. This experiment helped us to understand the predominant occurrence of hydrophobic interactions using urea that tends to prevent the dye aggregation. Similarly, urea was observed to break the interactions between polyglutamic acid and methylene blue held weakly through electrostatic and hydrophobic interactions, thus confirming the existence of PGA-MB complex in a very narrow range of high molecular weight polyglutamic acid concentration.

System gets much more complicated when the inducer of the dye's metachromasy undergoes conformational changes by itself. Polyglutamic acid is known to exist in several different forms depending on the surrounding conditions. Therefore, the probability of polyglutamic acid undergoing conformational changes can also not be denied. To further strengthen our hypothesis of charge and concentration dependent binding between polyglutamic acid and methylene blue, we have attempted to analyze this complexation with fluorescence spectroscopy and MD simulation data.

Fluorescence spectroscopy: Further elucidation of polymerdye binding mechanism was carried out using fluorescence spectroscopy. Experiments were carried out with three different concentrations of methylene blue $(6.25,12.5$ and $25 \mu \mathrm{M}$ methylene blue), out of which $12.5 \mu \mathrm{M}$ methylene blue was observed to be best suited for further polymer binding studies with respect to emission intensity. Excitation wavelength of $550 \mathrm{~nm}$ was fixed on the basis of the absorption spectra of methylene blue in the presence of polyglutamic acid for carrying out fluorescence spectroscopy as shown in Fig. 7a. A significant decrease in the fluorescence intensity of methylene blue was observed due to addition of polyglutamic acid in the concentration range of 0.0006-0.0066 $\mu \mathrm{M}$, which on further enhancement exhibited saturation beyond $0.012 \mu \mathrm{M}$. Emission and absorption spectra of the PGA-MB complex revealed the similar binding concentration range indicative of the changes occurring in the dye molecules arising due to interactions with polyglutamic acid. The fluorescence quenching data for the association of polyglutamic acid and methylene blue was analyzed using the Stern-Volmer equation.

$$
\frac{\mathrm{F}_{\mathrm{o}}}{\mathrm{F}}=1+\mathrm{K}_{\mathrm{SV}}[\mathrm{Q}]
$$

where $F_{o}$ and $F$ are the fluorescence intensities of the methylene blue in absence and presence of polyglutamic acid respectively 

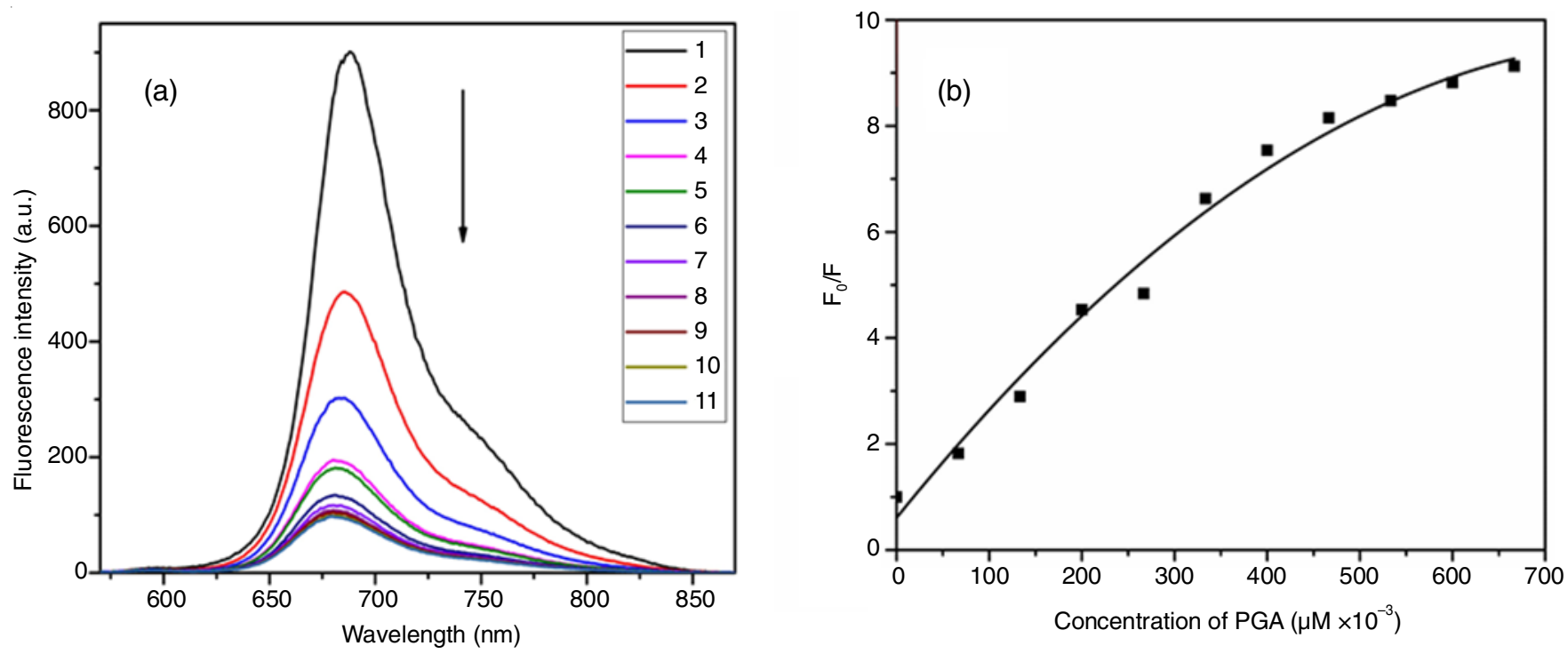

Fig. 7. (a) Emission spectra of methylene blue $(12.5 \mu \mathrm{M})$ only and with polyglutamic acid where the numerals signify the presence of increasing order of polyglutamic acid concentration from 0.0006 to $0.0066 \mu \mathrm{M}$; (b) Stern Volmer quenching plot for MB-PGA system in aqueous medium with $\lambda_{\max }$ at $550 \mathrm{~nm}$ showing negative deviation. $\mathrm{F}$ and $\mathrm{F}_{\mathrm{o}}$ represent fluorescence intensity with and without the quencher

and $\mathrm{Q}$ is the quencher (polyglutamic acid) concentration. The plot of $\mathrm{F}_{\mathrm{o}} / \mathrm{F}$ versus $[\mathrm{Q}]$ shows a negative deviation beyond a particular concentration of polyglutamic acid $(0.004 \mu \mathrm{M})$ as shown in Fig. 7b. However, the calculated slope of the linear region of the plot gives a $\mathrm{K}_{\mathrm{sv}}$ value of $1.59 \times 10^{-8} \mathrm{M}^{-1}$.

There are enough evidences available to prove that the basic dyes like methylene blue contain dimer species that are non-fluorescent as the higher molecular exciton level are optically active but the lower level is restricted [42]. D'mello et al. [18] mentioned the occurrence of metachromasy in dyes where the blue colour of the methylene blue in water solution was imparted due to monomers of the dye, whereas the change in the colour from blue to purple was attributed to the dimers, trimers and higher aggregated dye molecules. We have observed the similar colour change i.e. from maiden blue to purple on adding polyglutamic acid to the methylene blue solution.

Fluorescence quenching may arise due to molecular rearrangements, energy transfer and formation of ground-state complex, excited-state reactions and collisional quenching [43]. Environmental factors such as solvent polarity, concentration of quencher molecules and temperature also play crucial role in the whole process [30]. From the fluorescence data, it can be inferred that the interaction between polyglutamic acid and methylene blue molecules is a concentration dependent phenomenon. Fluorescence emission of methylene blue molecules was observed to be reduced significantly in presence of polyglutamic acid which acted as the quencher in present study. However on further increasing the concentration of polyglutamic acid beyond $0.6 \mu \mathrm{M}$, the quenching effect was observed to get diminished probably due to protection of dye molecules against quenching in presence of the macromolecule. Thus, polyglutamic acid was observed to accelerate the decay rate of methylene blue when it was in its excited state due to fluorescence. There is a possibility of presence of two types of fluorophores in the dye-polymer complex. One was the monomeric form of the dye and the other higher aggregate form which is more likely get masked in the polymeric chains of polyglutamic acid molecules. This is indicative of methylene blue aggregation of higher order where the fluorophore has lower accessibility to the quencher molecules. Similar pattern of trimer formation of dye in the same concentration range of polyglutamic acid was observed in UV-visible spectroscopy. Therefore, it is concluded from the spectroscopic data that it is a sensitive method to determine the polymer-dye complex in the lower concentration range as well.

Molecular dynamics (MD) simulation studies: In order to understand the molecular interactions leading to methylene blue aggregation and PGA-MB binding, we have carried out atomistic MD simulations of methylene blue solution both in presence and absence of polyglutamic acid. Due to the system size limitations of MD simulations, we needed to use much higher concentrations of methylene blue as compared to the experimental work ( 3 methylene blue molecules in a simulation box containing about 40,000 water molecules correspond to about $4 \mathrm{mM}$ ). Under these conditions the planar methylene blue molecules exist in a dynamic equilibrium between monomer, dimer and trimer species. It is noticed that in the presence of polyglutamic acid, the methylene blue aggregates are often strongly bound to the polyglutamic acid. The dimer and trimer forms of methylene blue were predominantly found near the polyglutamic acid surface. Fig. 8 shows representative snapshots of the dimer and trimer forms of methylene blue bound to the polyglutamic acid chain. Despite the electrostatic repulsion between the charged methylene blue molecules, they tend to aggregate in aqueous medium due to $\pi$ stacking and hydrophobic interactions [33]. The polyelectrolyte polyglutamic acid, which has a high local charge density, contributes to high ionic strength in the vicinity of the surface that would further screen the electrostatic repulsion between the methylene blue molecules and thus the hydrophobic attraction would win over the electrostatic repulsion. Thus, the higher order aggregates are promoted by the presence of polyglutamic acid. 

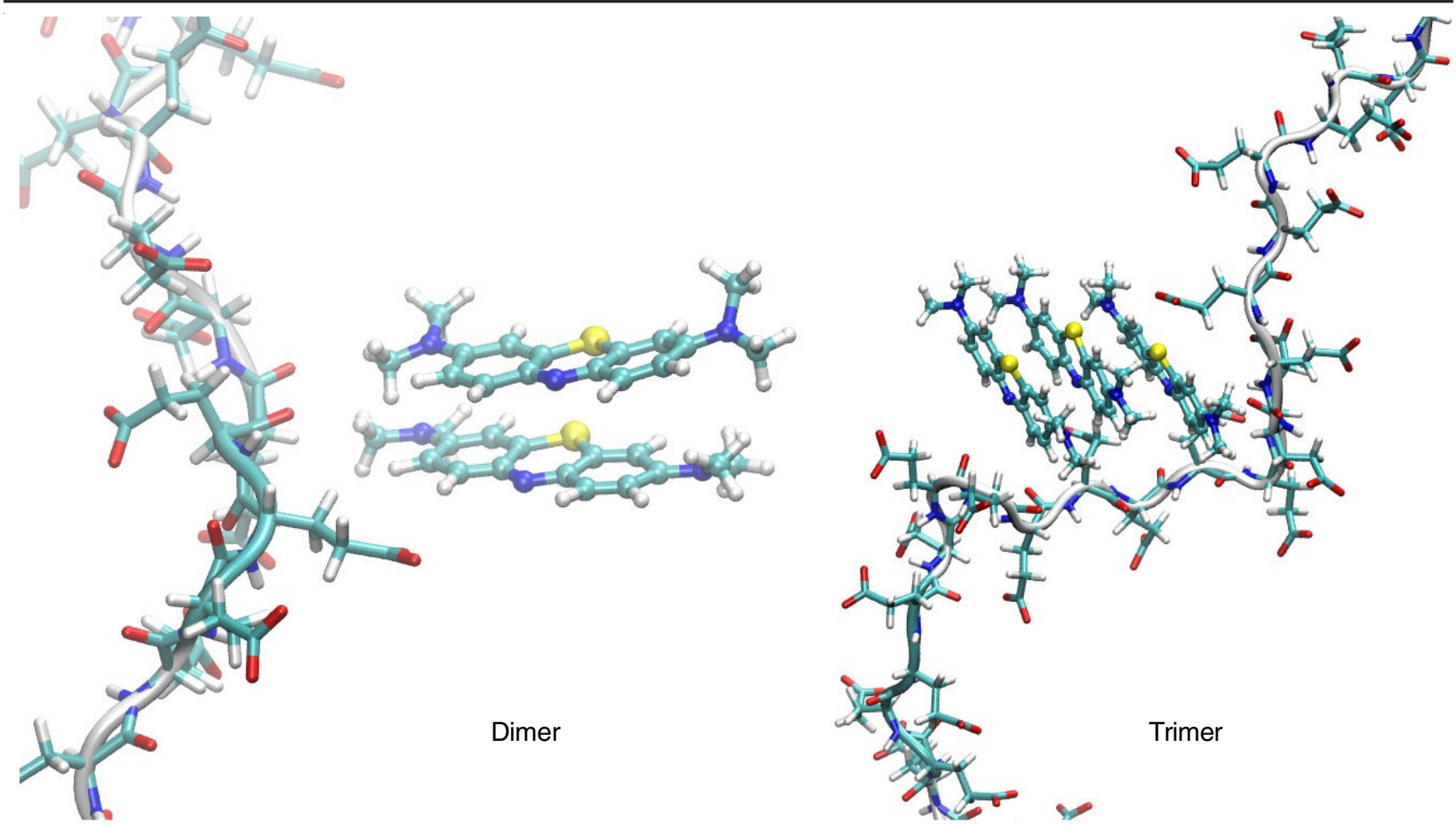

Fig. 8. Representative snapshots from MD simulation trajectories highlighting the formation of dimer and trimer states of methylene blue being bound to polyglutamic acid

We have simulated a solution of a single polyglutamic acid chain (containing 50 monomers) and three methylene blue molecules to understand how the presence of polyglutamic acid affects the aggregation behaviour of methylene blue. Cluster size distribution of methylene blue (Fig. 9) in presence and absence of polyglutamic acid revealed that the relative population of various aggregate sizes (dimer, trimer, etc.) strongly depend on the presence of polyglutamic acid as discussed above.

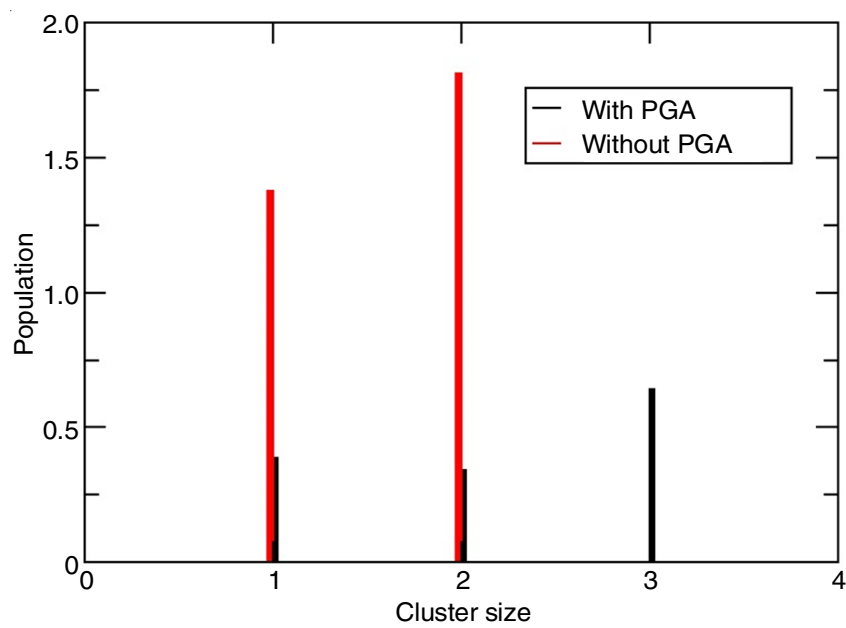

Fig. 9. Comparison of cluster (aggregate) size distribution of methylene blue with and without polyglutamic acid

The high affinity of methylene blue towards polyglutamic acid manifests into an altered aggregation profile of the methylene blue molecules. Thus, the increase in polyglutamic acid concentration would lead to change in the relative ratios of various aggregate sizes of methylene blue causing the metachromatic variations. The trimer population was found to be significantly higher in the presence of polyglutamic acid as compared to the dimer species. Thus, MD simulation studies confirm the hypothesis that the presence of polyglutamic acid can drastically alter the aggregation profile of methylene blue similar to the observations in the UV-visible studies.

Neutral red has been proposed in the previous studies [3] for screening polyglutamic acid producing microbes on agar plate. This triggered our interest to compare methylene blue with neutral red for binding with polyglutamic acid molecules. Therefore, the comparison between the binding affinity of methylene blue and neutral red with polyglutamic acid has been elucidated in terms of the distance profile between the dye and polyglutamic acid (Fig. 10). Although neutral red approaches polyglutamic acid surface quite frequently, it quickly detaches itself since the binding energy is significantly low due to the weaker electrostatic interactions between neutral neutral red and polyglutamic acid. Whereas, the methylene blue molecule binds irreversibly to the polyglutamic acid surface within $6 \mathrm{~ns}$ and does not detach within the simulation timescale. Clearly, cationic methylene blue has significantly higher binding affinity towards polyglutamic acid as compared to neutral red.

The Ramachandran plots [44] of polyglutamic acid were constructed from two-dimensional histograms (probability distribution) of the individual phi/psi angles of all residues calculated over the whole trajectory. The comparison of Ramachandran plots of polyglutamic acid in presence and absence of methylene blue as shown in Fig. 11a and b seems to exhibit a subtle change in the conformational distribution of polyglutamic acid. The normalized histogram indicate 

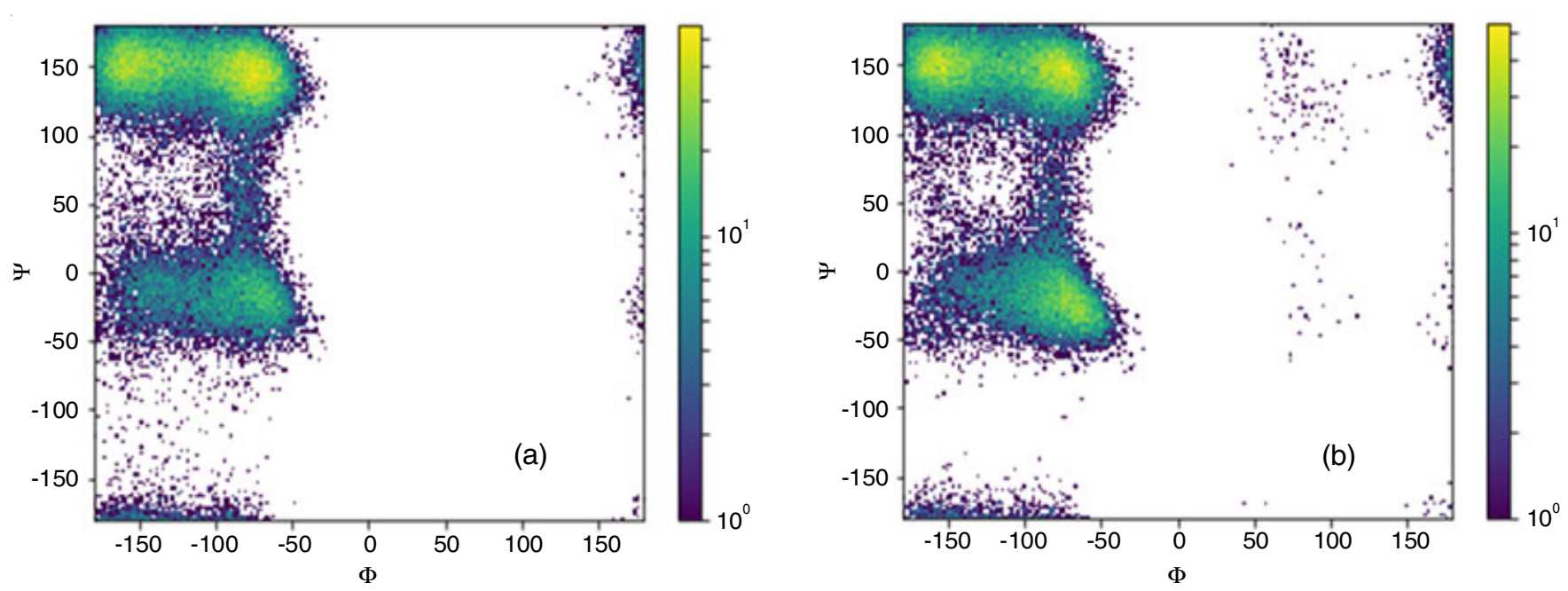

Fig. 11. Comparison of Ramachandran plot of polyglutamic acid in the presence (a) and absence (b) of methylene blue depicting subtle conformational changes in polyglutamic acid induced by methylene blue. The probability density near the helical region is reduced in the presence of methylene blue (a)

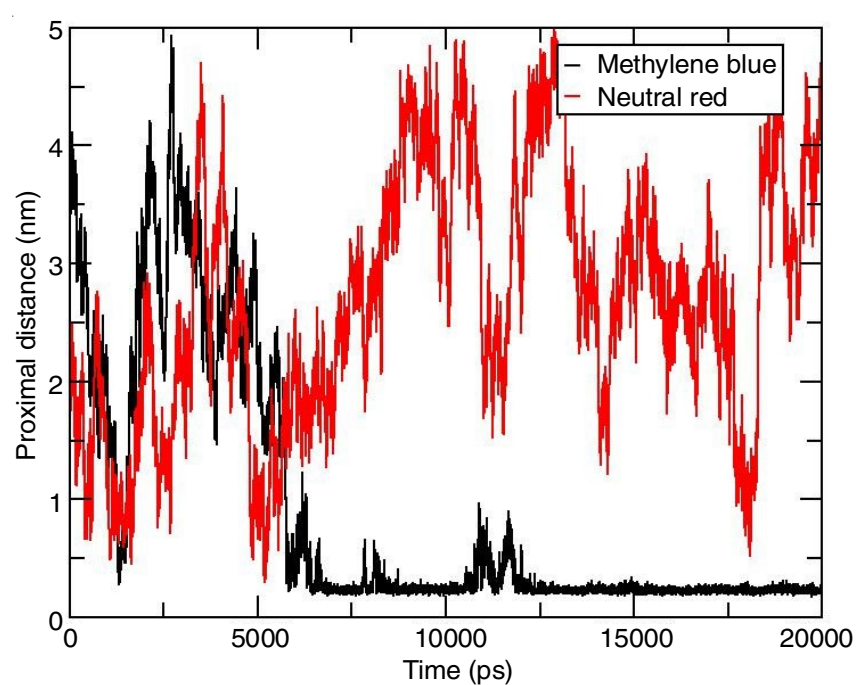

Fig. 10. Time evolution of proximal distance between polyglutamic acid and dye (methylene blue and neutral red): Small values of this distance would indicate binding between polyglutamic acid and dye

slightly lower population density in the $\alpha$-helical region of Ramachandran plot in the presence of methylene blue. This would imply a marginal increase in the relative population of the $\beta$-sheet region of the plot. However, it is to be noted that the Ramachandran plot involves the conformational distribution only at a single residue level. We do not observe actual formation of extended $\beta$-sheet in our simulations, which would require formation of parallel/anti-parallel $\mathrm{H}$-bonds between the backbone atoms.

We must clarify here that the specific conditions of the simulations and experimental studies vary by a large margin. For example, the simulation study deals with a rather small 50-mer of polyglutamic acid, whereas in the experiments the polyglutamic acid chains can be as large as $100,000 \mathrm{kDa}$. The effective concentrations of both methylene blue and polyglutamic acid are significantly higher in the simulations as well. Nevertheless, the simulation results clearly provide validation to the general hypotheses that (i) the presence of polyglutamic acid can alter the aggregation profile of the methylene blue molecules and (ii) presence of methylene blue can alter the conformational state of polyglutamic acid.

\section{Conclusion}

All the analytical tools used in the study revealed the affinity of methylene blue molecules towards polyglutamic acid. The negative charges on polyglutamic acid molecules were observed to play a crucial role in binding with positive charges on methylene blue molecules and in turn these dye molecules were observed to have a tendency to undergo aggregation. UV-visible absorption studies in aqueous medium revealed the tendency of methylene blue molecules to undergo aggregation in presence of polyglutamic acid as indicated by the occurrence of blue shift in the spectra. Interaction of polyglutamic acid and methylene blue was further substantiated by the fluorescence quenching effect on methylene blue in presence of polyglutamic acid. Dye aggregation at higher concentrations of polyglutamic acid was further confirmed by the negative deviation of the Stern-Volmer plot. Molecular dynamics simulation studies also indicated the similar phenomenon of the both the molecules undergoing structural and conformational changes due to each other's presence. Methylene blue was observed to have higher binding affinity for polyglutamic acid when compared with neutral red dye indicating the strong role of positive charges on methylene blue molecules. In the current work we thus propose the involvement of multiple forms of interactions like electrostatic, hydrophobic and stacking along with the concentration dependent occurrence. Mechanism of interaction of PGA-MB system can further be used for designing the quantification methods for the biopolymer production. The sensitivity of the assay to detect the polyglutamic acid with the concentration as low as $1.9 \mathrm{nM}$ can be exploited using UV-visible Spectrophotometer. UV-visible approach to qualitatively analyse the presence of polyglutamic acid in the fermentation broth directly can significantly reduce the time taken for the analysis of this biopolymer during primary screening of polyglutamic acid producing bacteria in liquid broth. 


\section{ACKNOWLEDGEMENTS}

This work has been funded by Tata Chemicals Limited, Pune, India. Part of the work was also supported by National Chemical Laboratory (NCL), Pune and Indian Institute of Science Education and Research (IISER), Pune. We thank the Confederation of Indian Industry (CII) to support P.M. Chatterjee and D.P. Tiwari through Prime Minister Fellowship Scheme for Doctoral research, a public-private partnership between Science and Engineering Research Board (SERB). Authors also thank Manipal University for registering the above mentioned $\mathrm{PhD}$ scholars to pursue their research work on $\gamma$-polyglutamic acid.

\section{CONFLICT OF INTEREST}

The authors declare that there is no conflict of interests regarding the publication of this article.

\section{REFERENCES}

1. I.R. Khalil, V.U. Irorere, I. Radecka, A.T.H. Burns, M. Kowalczuk, J.L. Mason and M.P. Khechara, Materials, 9, 28 (2016); https://doi.org/10.3390/ma9010028.

2. H. Kubota, Y. Nambu and T. Endo, J. Polym. Sci. A Polym. Chem., 34, 1347 (1996); https://doi.org/10.1002/(SICI)1099-0518(199605)34:7<1347::AIDPOLA24>3.0.CO;2-8.

3. I. Bajaj and R. Singhal, Bioresour. Technol., 102, 5551 (2011); https://doi.org/10.1016/j.biortech.2011.02.047.

4. W. Zeng, Y. Lin, Z. Qi, Y. He, D. Wang, G. Chen and Z. Liang, Appl. Microbiol. Biotechnol., 97, 2163 (2013); https://doi.org/10.1007/s00253-013-4717-0.

5. Y. Ogawa, F. Yamaguchi, K. Yuasa and Y. Tahara, Biosci. Biotechnol. Biochem., 61, 1684 (1997); https://doi.org/10.1271/bbb.61.1684.

6. G.A. Birrer, A.M. Cromwick and R.A. Gross, Int. J. Biol. Macromol., 16, 265 (1994); https://doi.org/10.1016/0141-8130(94)90032-9.

7. P.M. Chatterjee, S. Datta, D.P. Tiwari, R. Raval and A.K. Dubey, Appl. Biochem. Biotechnol., 185, 270 (2018); https://doi.org/10.1007/s12010-017-2654-6.

8. J.A. Bergeron and M. Singer, J. Cell Biol., 4, 433 (1958); https://doi.org/10.1083/jcb.4.4.433.

9. F. Yamaguchi, Y. Ogawa, M. Kikuchi, K. Yuasa and H. Motai, Biosci. Biotechnol. Biochem., 60, 255 (1996); https://doi.org/10.1271/bbb.60.255.

10. B.S. Inbaraj and B.H. Chen, Bioresour. Technol., 102, 8868 (2011); https://doi.org/10.1016/j.biortech.2011.06.079.

11. B.S. Inbaraj, C.P. Chiu, G.H. Ho, J. Yang and B.H. Chen, J. Hazard. Mater, 137, 226 (2006); https://doi.org/10.1016/j.jhazmat.2006.01.057.

12. P.A. Tafulo, R.B. Queirós and G. González-Aguilar, Spectrochim. Acta A Mol. Biomol. Spectrosc., 73, 295 (2009); https://doi.org/10.1016/i.saa.2009.02.033.

13. B. Liu, L. Wen, K. Nakata, X. Zhao, S. Liu, T. Ochiai, T. Murakami and A. Fujishima, Eur. Chem. J., 18, 12705 (2012); https://doi.org/10.1002/chem.201200178.

14. S.B. Mahmoud, A.H. Hamzaoui and W. Essafi, Meditter. J. Chem., 5, 493 (2016); https://doi.org/10.13171/mjc54/01606170930/essafi.

15. L. Antonov, G. Gergov, V. Petrov, M. Kubista and J. Nygren, Talanta, 49, 99 (1999); https://doi.org/10.1016/S0039-9140(98)00348-8.

16. D. Heger, J. Jirkovski and P. Klan, J. Phys. Chem. A, 109, 6702 (2005); https://doi.org/10.1021/jp050439j.

17. K. Patil, R. Pawar and P. Talap, Phys. Chem. Chem. Phys., 2, 4313 (2000); https://doi.org/10.1039/B005370H
18. A.X. D'mello, T.V. Sylvester, V. Ramya, F.P. Britto, P.K. Shetty and S. Jasphin, Int. J. Adv. Health Sci., 2, 12 (2016).

19. R.B. McKay and P.J. Hillson, Faraday Soc., 61, 1800 (1965); https://doi.org/10.1039/tf9656101800.

20. M.J. Abraham, T. Murtola, R. Schulz, S. Páll, J.C. Smith, B. Hess and E. Lindahl, SoftwareX, 1-2, 19 (2015); https://doi.org/10.1016/j.softx.2015.06.001.

21. K. Lindorff-Larsen, S. Piana, K. Palmo, P. Maragakis, J.L. Klepeis, R.O. Dror and D.E. Shaw, Proteins, 78, 1950 (2010); https://doi.org/10.1002/prot.22711.

22. W.L. Jorgensen, J. Chandrasekhar, J.D. Madura, R.W. Impey and M.L. Klein, J. Phys. Chem., 79, 926 (1983); https://doi.org/10.1063/1.445869.

23. G. Bussi, D. Donadio and M. Parrinello, J. Phys. Chem., 126, 014101 (2007); https://doi.org/10.1063/1.2408420.

24. M. Parrinello and A. Rahman, J. Appl. Phys., 52, 7182 (1981); https://doi.org/10.1063/1.328693.

25. U. Essmann, L. Perera, M.L. Berkowitz, T. Darden, H. Lee and L.G. Pedersen, J. Chem. Phys., 103, 8577 (1995); https://doi.org/10.1063/1.470117.

26. B. Hess, H. Bekker, H.J.C. Berendsen and J.G. Fraaije, J. Comput. Chem., 18, 1463 (1997) https://doi.org/10.1002/(SICI)1096-987X(199709)18:12<1463::AIDJCC4>3.0.CO;2-H.

27. E. Fosso-Kankeu and L. Simelane, Kinetic Study of Methylene Blue Removal by Gram-Negative and Gram-Positive Bacteria, International Conference on Chemical, Mining and Metallurgical Engineering CMME'2013, Johannesburg, South Africa (2013).

28. F. Ogata, N. Nagai and N. Kawasaki, Chem. Pharm. Bull. (Tokyo), 65, 268 (2017); https://doi.org/10.1248/cpb.c16-00827.

29. B.C. Myhr and J.G. Foss, Biopolymers, 4, 949 (1966); https://doi.org/10.1002/bip.1966.360040810.

30. M.K. Goftar, K. Moradi and N.M. Kor, Eur. J. Exp. Biol., 4, 72 (2014).

31. E.K. Golz and D.A. Vander Griend, Anal. Chem., 85, 1240 (2013); https://doi.org/10.1021/ac303271m

32. S. Jockusch, N.J. Turro and D.A. Tomalia, Macromolecules, 28, 7416 (1995); https://doi.org/10.1021/ma00126a020.

33. E. Braswell, J. Phys. Chem., 72, 2477 (1968); https://doi.org/10.1021/j100853a035.

34. J.S. Moore, G.O. Phillips, D.M. Power and J.V. Davies, J. Chem. Soc. A, 1155 (1970); https://doi.org/10.1039/J19700001155.

35. E. Coates, Color. Technol., 85, 355 (1969).

36. Y. Hu, T. Guo, X. Ye, Q. Li, M. Guo, H. Liu and Z. Wu, Chem. Eng. J., 228, 392 (2013); https://doi.org/10.1016/j.cej.2013.04.116.

37. B. Heyne, Photochem. Photobiol. Sci., 15, 1103 (2016); https://doi.org/10.1039/C6PP00221H.

38. D. Sarkar, P. Das, S. Basak and N. Chattopadhyay, J. Phys. Chem. B, 112, 9243 (2008); https://doi.org/10.1021/jp801659d.

39. S. Datta, C. Mukhopadhyay and S.K. Bose, Bull. Chem. Soc. Jpn., 76, 1729 (2003) https://doi.org/10.1246/bcsj.76.1729.

40. A. Wallqvist, D.G. Covell and D. Thirumalai, J. Am. Chem. Soc., 120, 427 (1998); https://doi.org/10.1021/ja972053v.

41. P. Mukerjee and A.K. Ghosh, J. Am. Chem. Soc., 92, 6419 (1970); https://doi.org/10.1021/ja00725a006.

42. G.N. Lewis, O. Goldschmid, T.T. Magel and J. Bigeleisen, J. Am. Chem. Soc., 65, 1150 (1943); https://doi.org/10.1021/ja01246a037.

43. J.R. Lakowicz, Quenching of Fluorescence in Principles of Fluorescence Spectroscopy, Springer, Boston: USA, edn 3 (2006).

44. R.V. Mannige, PeerJ, 5, e3327 (2017); https://doi.org/10.7717/peerj.3327. 